\title{
Nitinol spring rod dynamic stabilization system and Nitinol memory loops in surgical treatment for lumbar disc disorders: short-term follow up
}

\author{
Young-Soo Kim, M.D., Ph.D., ${ }^{1,2}$ Ho-Yeol Zhang, M.D., Ph.D., ${ }^{2,3}$ \\ Byung-Jin Moon, M.D., ${ }^{1}$ Kyung-Woo Park, M.D., PH.D., ${ }^{1}$ KyU-Yeul Ji, M.D., ${ }^{1}$ \\ Won-Chang LeE, M.D., ${ }^{1}$ KYU-Sung OH, M.D., ${ }^{1}$ GWON-Ui RYU, M.D., 1 \\ AND DANIEL H. KIM, M.D. ${ }^{4}$ \\ ${ }^{1}$ Department of Neurosurgery, Kwang-Hye Spine Hospital, Seoul, Korea; \\ ${ }^{2}$ Department of Neurosurgery, Yonsei University, Seoul, Korea; \\ ${ }^{3}$ Department of Neurosurgery, National Health Insurance Corporation Ilsan Hospital, \\ Goyang, Korea; and ${ }^{4}$ Department of Neurosurgery, Ochsner Medical Center, \\ New Orleans, Louisiana
}

\begin{abstract}
Object. The purpose of this study was to analyze the usefulness of the BioFlex, a Nitinol spring rod dynamic stabilization system, and the Nitinol shape memory loop (KIMPF-DI Fixing System) as a posterior dynamic stabilization system in surgery for low-back pain.

Methods. The 103 patients who underwent treatment with the BioFlex system were divided into two groups: Group 1, dynamic stabilization with or without posterior lumbar interbody fusion (PLIF); and Group 2, rigid fixation (PLIF + BioFlex system only). A total of 66 segments were treated with only the BioFlex system; in these the preoperative range of motion $(\mathrm{ROM})$ was $10.0 \pm 4.3^{\circ}$, which changed to $4.1 \pm 1.9^{\circ}$ after surgery. Adjacent-segment ROM changed from $8.4 \pm 3.4^{\circ}$ to $10.7 \pm 3.2^{\circ}$ in Group 1 and from $6.5 \pm 3.2^{\circ}$ to $10.5 \pm 4.6^{\circ}$ in Group 2 postoperatively. A total of 110 segments received both BioFlex and PLIF, with a fusion rate of $90.0 \%$. The visual ana$\log$ scale score for back pain improved from $7.3 \pm 3.1$ to $1.4 \pm 1.8$ in Group 1 and from $7.4 \pm 2.4$ to $2.1 \pm 2.3$ in Group 2. The Oswestry Disability Index improved from $35.2 \pm 6.4$ to $12.1 \pm 4.5$ in Group 1 and from $37.8 \pm 5.7$ to $13.6 \pm 4.2$ in Group 2. (The ROM and assessment scores expressed are the mean \pm standard deviation.)

The 194 patients in whom Nitinol memory loops were implanted were analyzed based on the preoperative and 1year postoperative ROM of each lumbar segment. The change of ROM in looped segments treated with PLIF was significantly reduced, but the change of ROM in looped segments without PLIF was not significant. The change of ROM at the segment adjacent to the loop was not significant, and the change of kyphosis reflected a slight recovery.

Conclusions. The Nitinol BioFlex dynamic stabilization system can achieve stabilization and simultaneously allow physiological movement, which can in turn decrease the degeneration of adjacent segments. When used with PLIF, the fusion rate can be expected to increase. The flexible Nitinol shape memory loop, a posterior dynamic stabilization device, is an adequate tension band that displays strength similar to the posterior ligamentous structures. In combination with PLIF at the main lesion, the BioFlex system or the Nitinol memory loop can provide posterior dynamic stabilization to the transitional upper or lower segments, enhance the fusion rate, reduce the adjacentsegment degeneration, and provide dynamic stabilization of the spine.
\end{abstract}

\section{KEY WORDS • dynamic stabilization • Nitinol • semirigid spring rod system • shape memory loop}

$\mathrm{S}$ PINAL fusion with rigid fixation has been the mainstay for surgical treatment of low-back pain or instability. Nevertheless, despite the improvement in radiologically confirmed fusion rates, acceleration of adjacent-segment degeneration leads to "topping off" or "bottoming off" phenomena. Other complications such as hardware failure, fracture of the vertebral body or pedicle, and loss of lumbar lordosis have been reported.

The goal in producing the ideal stiffness after spine surgery is to obtain postoperative stability, deformity correction, and more physiological bone fusion, and to avoid

Abbreviations used in this paper: FBSS = failed-back surgery syndrome; PLIF = posterior lumbar interbody fusion; $\mathrm{ROM}=$ range of motion. mechanical failure, deterioration of the adjacent segment, and stiffness of the back. These concepts have changed the type of operations being performed from rigid fixation to soft fixation or dynamic stabilization. We proposed combined fixation in 1997.,8 This concept is a combination of rigid fixation (PLIF with interbody cage with or without a rigid pedicle screw) at the main diseased segment, and soft fixation at the adjacent transitional segment. This soft fixation can reduce the compensatory hypermotion stress associated with a rigidly fixed segment and can preserve the stabilized motion.

Nitinol is an alloy of nickel and titanium that belongs to a class of materials called shape memory alloys. It was invented in 1962 by researchers at the US Naval Ordnance Laboratory. ${ }^{10}$ The name represents its elemental compo- 

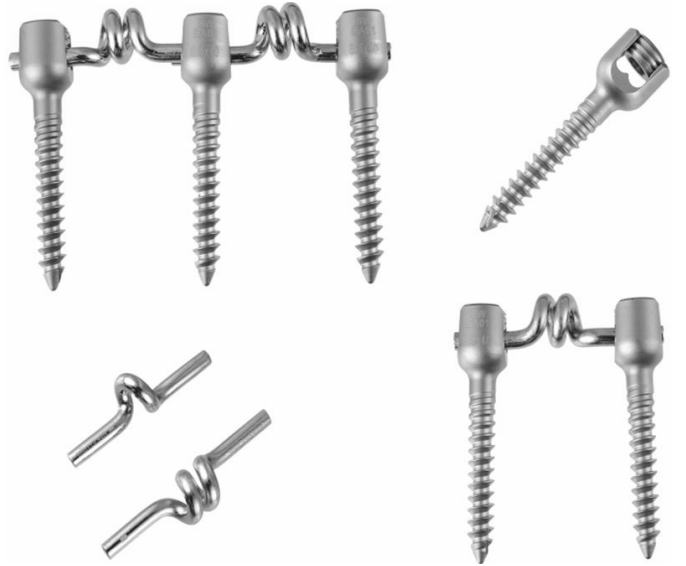

FIG. 1. Photographs showing the components of the BioFlex dynamic stabilization system, which is composed of titanium screws and Nitinol rods. The rods are of two types, one with a single turn forming the coil and one with two turns in the coil. In the upper right corner the photograph shows the BioFlex screw head design. Its two grooves enable insertion of two rods per segment in cases in which more than one segment is stabilized. (Reprinted with permission from Bio-Spine Corp.)

nents and place of origin: $\mathrm{Ni}$ and $\mathrm{Ti}$ are the chemical symbols for nickel and titanium, and "nol" stands for the Naval Ordnance Laboratory where it was discovered. The Nitinol implant has various characteristics such as high elasticity and high tensile force, flexibility (below $10^{\circ} \mathrm{C}$ ) or rigidity (above $30^{\circ} \mathrm{C}$ ) according to temperature change, and biological compatibility.

A new dynamic stabilization system (BioFlex System; Bio-Spine) consisting of titanium pedicle screws and a Nitinol rod (American Society for Testing and Materials F2063) was developed in Korea. The rod diameter is $4 \mathrm{~mm}$, with coiled shapes of one and two turns, which brings physiological stability during flexion, extension, and lateral bending. A design-specific advantage also allows ease in implantation and connectability for each segment (Fig. 1). ${ }^{9}$

The shape memory loop implant (KIMPF-DI Fixing System; CJSC KIMPF Co.) is made from Nitinol (Fig. 2). It can be used as a posterior tension band, mostly in de-
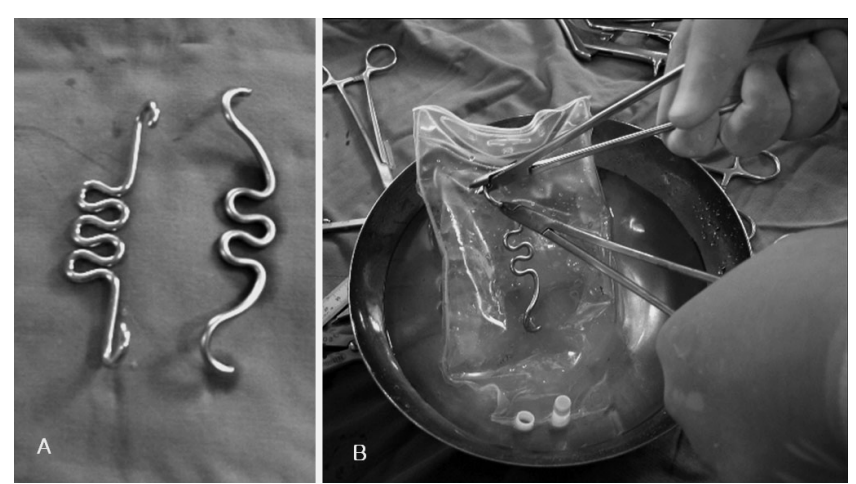

FIG. 2. Photographs showing the Nitinol shape memory loop before and after deformation. A: On the left is a loop in its original shape and on the right is one in a deformed state. B: Deformation of Nitinol shape memory loop after immersion in $10^{\circ} \mathrm{C}$ saline. compressive laminectomy cases. We have used these implants with or without PLIF for the last 2 years, and in this paper we have analyzed these systems to assess their indications, surgical methods, postoperative results, and complications. The ROM, angles, and assessment scores are expressed as the mean $\pm \mathrm{SD}$.

\section{Clinical Material and Methods}

\section{Patient Population}

The mean age of the patients in BioFlex Groups 1 and 2 was 49.9 years (range 18-83 years) and 55.65 years (range 20-87 years), respectively. In Group 1 there were 13 men and 33 women, and in Group 2 there were 12 men and 45 women. The mean follow-up period for patients in Group 1 was 9.3 months (range 6-17 months) and in Group 2 it was 10.6 months (range 6-17 months).

\section{BioFlex System}

The BioFlex system was used from February 15, 2005 through the end of March 2006 in 164 patients. A retrospective review was conducted in the 103 patients who were available for follow-up assessment for more than 6 months. All patients had presented with symptoms of disabling back pain with or without leg pain, and had experienced no improvement after 6 weeks or more of conservative treatment. Surgery was performed at two hospitals (Kwanghye Spine Hospital in Seoul and National Health Insurance Corporation Ilsan Hospital in Goyang, Korea) by five surgeons with more than 10 years of pedicle screw fixation experience.

The 103 patients were divided into two groups for study. Group 1 was the dynamic stabilization group, which included 46 patients who were treated with just the BioFlex system after wide laminectomy with or without discectomy, or $360^{\circ}$ fixation with an interbody cage and the BioFlex device at the main diseased segment and BioFlex stabilization at the adjacent transitional segments (Fig. 3). Group 2 was the rigid fixation group, which included 57 patients who received $360^{\circ}$ fixation with an interbody cage implanted for PLIF and BioFlex fixation at the diseased segments only (Fig. 4).

The surgical indication for this procedure is identical to that for the commercially common pedicle screws, with a relative contraindication of active infection. In this series, most of the patients had degenerative lumbar disc disease; only three patients in Group 1 displayed lumbar bursting fracture. The most common disorder was spondylolytic spondylolisthesis (in 46 patients), followed by 26 with degenerative spondylolisthesis, 12 with degenerative spinal canal stenosis, nine with chronic degenerative herniated lumbar disc, seven with FBSS, and three with trauma (Table 1).

\section{Nitinol Memory Loop}

The Nitinol memory loop system was used between August 2004 and September 2005 in a total of 194 patients (male/female ratio 69:125, mean age $60.77 \pm 10.34$ years) with degenerative spinal diseases or osteoporotic compression fractures. The 194 patients were followed up over a 1-year period. The pertinent records for these pa- 


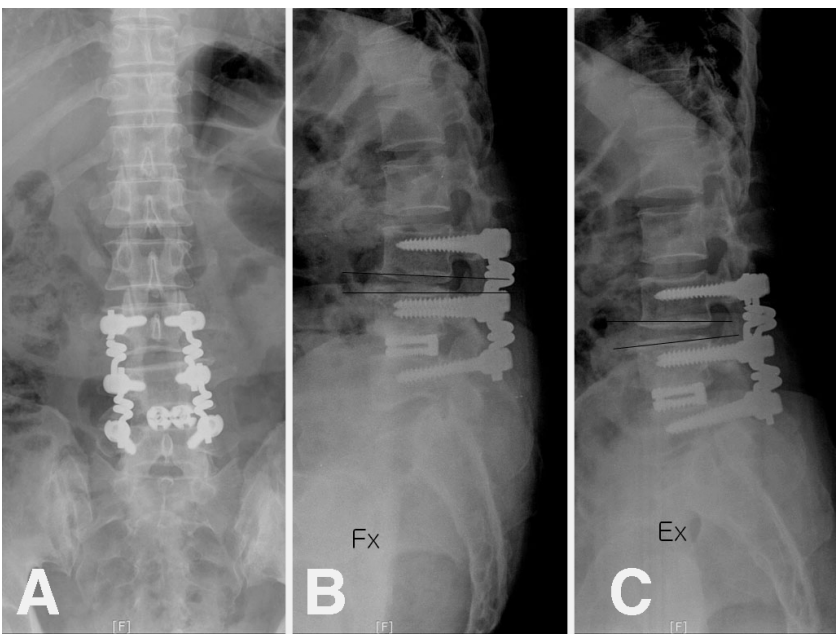

FIG. 3. Plain $\mathrm{x}$-ray films showing instrumentation in place in a patient in Group 1 (dynamic stabilization). The main diseased region was fused using PLIF and BioFlex fixation, and the degenerative adjacent segment was fixated using the BioFlex system.

tients were then retrospectively reviewed and the demographic data, surgical details, pre- and postoperative images, and the scores according to the functional scales of Prolo et al. ${ }^{12}$ were analyzed for each patient.

Our surgical indications for the application of shape memory loop were as follows: degenerative stenosis with or without disc herniation, posterior stabilization after PLIF, posterior stabilization after decompressive laminectomy, correction of degenerative kyphoscoliosis, compression fracture with kyphosis, degenerative spondylolisthesis, prevention of adjacent-segment instability, injury of posterior ligamentous structures, and repeated operation for FBSS.
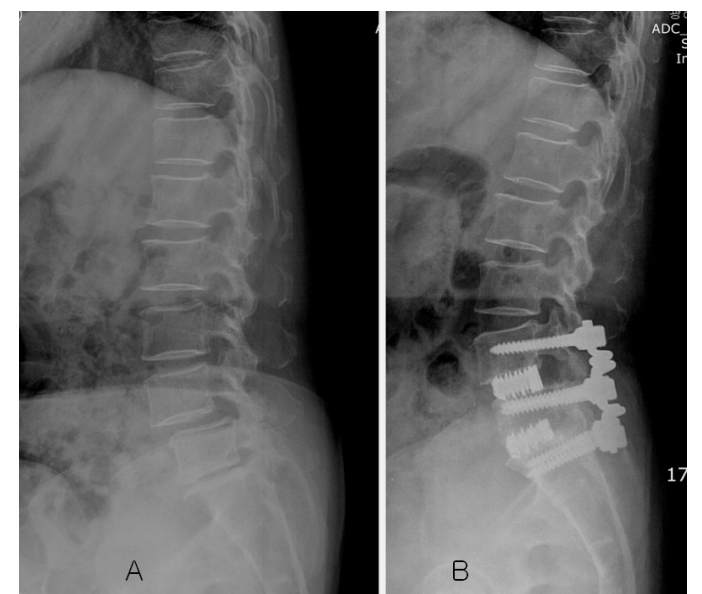

FIG. 4. Plain $\mathrm{X}$-ray films showing instrumentation in place in a patient in Group 2 (rigid fixation); PLIF and BioFlex fixation were performed at the same levels. A: Preoperative lateral plain radiographic view obtained in a 47-year-old man showing degenerative spondylolisthesis at L4/5 and narrowed disc space at L5/S1. B: Postoperative lateral view showing the instrumentation.

\section{Surgical Technique}

BioFlex System. Most patients received epidural anesthesia, and for those with three or more segments to be treated, surgery was performed after induction of general anesthesia. A Wilson frame was used for patient positioning, and sufficient abdominal decompression was applied to reduce blood loss. The usual posterior approach using a midline incision was performed. For the main diseased segment, the Profix (Phil Med. Co.) expandable or cylindrical cages were used for interbody fusion. The titanium screw was inserted according to the usual method. The available rod lengths were 40 to $100 \mathrm{~mm}$ by $5-\mathrm{mm}$ increments, one per segment insertion, without the need for cutting. Rods are also available for left and right sides, with the coil rotation different for each side. There are two grooves in the screw head; one groove for each rod, and the setscrew must be secured into the set housing after insertion of the rods (Fig. 1, upper right corner). If the insertion is in any way difficult, the rod is immersed in cold water, thereby making the device flexible and easy to handle. The rod recovers its shape at body temperature.

Nitinol Memory Loop. This technique is designed for the surgical treatment of L4/5 stenosis accompanied by L3/4 mild spondylotic stenosis (Figs. 2 and 5). After receiving epidurally or endotracheally administered anesthetic agents, the patient lies on a Wilson frame in the prone position. A midline linear incision and exposure are made from the L-3 to L-5 lamina. An L-4 laminectomy and removal of the ligamentum flavum of L4/5 are performed. The distance between the L-3 upper lamina and L-5 lower lamina will be shortened after loop application. If any ligamentum flavum remains during this shortening, it can play the role of the dorsal compressed mass of the dural sac. Therefore, complete removal of the ligamentum flavum is important. Discectomy and the PLIF procedure on L4/5 are performed in the usual fashion.

A very minimal focal skeletonization of the lamina or spinous processes of L-3 at its upper end and L-5 at its lower end is performed and a determination of where to apply the fixating parts is made. Template measuring instruments are used to calculate the exact size of the memory loop. When loop application between two motion seg-

TABLE 1

Disease classification in 103 patients treated with the BioFlex system*

\begin{tabular}{lccc}
\hline \hline & \multicolumn{3}{c}{ No. of Patients (\%) } \\
\cline { 2 - 4 } Disease & Group 1 & Group 2 & Total \\
\hline spondylolytic spondylolisthesis & $18(39.1)$ & $28(49.1)$ & $46(44.7)$ \\
degenerative spondylolisthesis & $11(23.9)$ & $15(26.3)$ & $26(25.2)$ \\
degenerative stenosis & $5(10.9)$ & $7(12.3)$ & $12(11.7)$ \\
chronic degenerative disc & $4(8.7)$ & $5(8.8)$ & $9(8.7)$ \\
FBSS or recurrent HLD & $5(10.9)$ & $2(3.5)$ & $7(6.8)$ \\
trauma & $3(6.5)$ & $0(0.0)$ & $3(2.9)$ \\
total & $46(100.0)$ & $57(100.0)$ & $103(100.0)$ \\
\hline
\end{tabular}

* Patients in Group 1 underwent dynamic stabilization, and those in Group 2 were treated with rigid fixation. Abbreviation: HLD = herniated lumbar disc. 

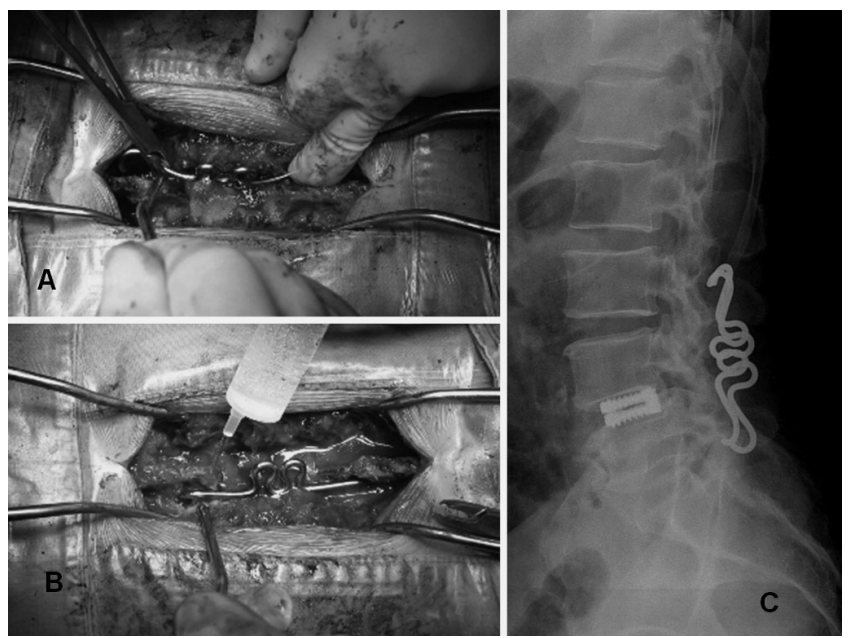

FIG. 5. A: Intraoperative photograph showing the deformed loops being placed by one hook behind the lamina arch of one vertebra, after which the second hook of the loop is placed behind the lamina arch of the next vertebra. B: Intraoperative photograph obtained after installation of a loop, showing irrigation with heated (up to $35-45^{\circ} \mathrm{C}$ ), sterilized physiological saline, which causes the implant to regain its initial form, fixating the diseased segment of the spine. C: Postoperative lateral $\mathrm{x}$-ray film showing the PLIF L4/5 and memory loop fixation from the L-3 to L-5 laminae.

ments is needed, we always use the 1.5- to 2-cm loop, which is smaller than the distance between the two laminae or between two spinous processes. The supine position and laminectomy cause the disruption of lumbar lordosis and posterior ligamentous structures. This leads to the pseudolengthening of the posterior column. If we use a loop the same size as the distance between the two laminae in the operating field, it can become loosened and dislodged.

After selection of the proper fixating loop, it is lifted by a forceps and cooled in sterilized physiological saline at 5 to $15^{\circ} \mathrm{C}$ for at least 30 seconds (Fig. 2). The loop holder is then used to deform the device before it is implanted. The deformed loops are placed by one hook behind the lamina arch of the vertebra (or spinous process) so that the plane of the loops is parallel to the spinal alignment, then the second hook of the loop is placed behind the lamina arch of another vertebra (or spinous process).

After installation of a loop, the implant is irrigated with sterile physiological saline heated to between 35 and $45^{\circ} \mathrm{C}$, which allows the implant to regain its initial form, thus fixing the diseased segment of the spine. Postimplantation photographs and an x-ray film with the implant in place are shown in Fig. 5.

\section{Clinical and Radiological Assessments}

BioFlex System. Patients were checked for improvements in clinical symptoms and development of complications, and their x-ray films obtained before and after surgery were analyzed. The ROM had been measured in the sagittal plane rotation of the functional spinal unit on dynamic (flexion-extension) lateral radiographs. Sagittal plane rotation represents the difference between the Cobb measurements taken in flexion and extension. The ROM was measured in patients in Group 1 in whom the fusion was performed (PLIF and BioFlex fixation) and the BioFlex system applied on the adjacent segments along with fusion, and the findings were compared with the ROM of the adjacent segments in all patients in Groups 1 and 2. For evaluation of postoperative sagittal lordosis in Group 1 , the lumbosacral angle in the lumbar lateral radiographic view was measured. The lumbosacral angle is defined as the angle between the lines penetrating the center of L5 and L-3, and L-5 and L-1.

All segments treated with PLIF were assessed whether or not the fusion was achieved, which means (as the definition of fusion states) the presence of a bone bridge in the side of the cage, or less than $5^{\circ}$ of movement on lateral flexion-extension views, and the absence of radiolucencies around the cage as well as absence of cage migration. Radiographic evaluation was performed before and after the operation and every 3 months thereafter. To compare the pre- and postoperative findings, each estimate was compared using the paired t-test. Back and leg pain were measured according to a 10-point visual analog scale before surgery and postoperatively at 3-month intervals. Disability was assessed using the Oswestry Disability Index before surgery and postoperatively at 3-month intervals.

Shape Memory Loop. Radiological evaluations of the ROM of the discs were performed preoperatively and 1 year postoperatively. Upper- and lower-segment ROM of the looped segments and in the looped levels were evaluated. We also determined the kyphotic angle in cases of kyphotic deformity. For this assessment, the angle was measured between the upper endplate of the uppermost vertebra undergoing looping and the lower endplate of the lowermost vertebra being treated in this fashion (Fig. 6). The clinical outcome was assessed using the Prolo scale at the 1-year follow-up visit, and complications were evaluated.
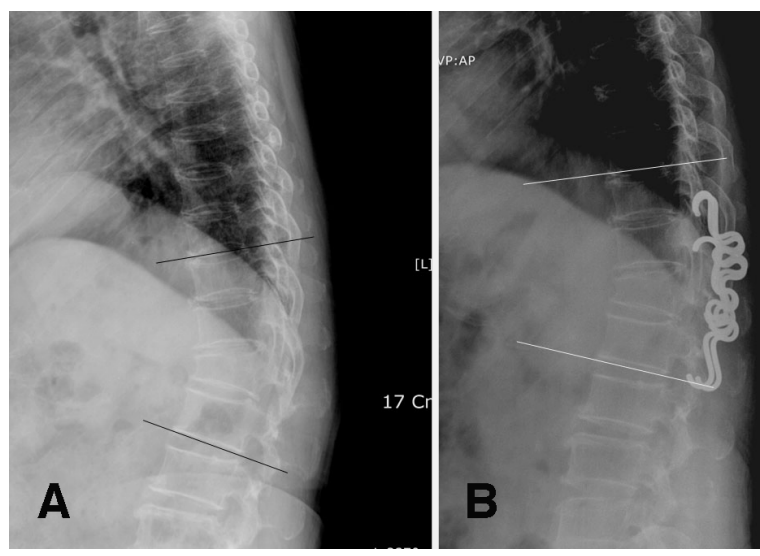

FIG. 6. Lateral x-ray films obtained pre- and postoperatively, showing an old compression fracture at L-1 in a patient with kyphosis who was treated with loop fixation. The kyphotic angle was measured between the upper endplate (T-10) of the uppermost vertebra undergoing looping and the lower endplate (L-2) of the lowermost vertebra treated with looping. 


\section{Results}

\section{BioFlex System}

In Group 1, 28 of the patients who were treated solely with the BioFlex system underwent fixation in a single segment, in 16 patients two segments were involved, and in two patients three segments were fixed among the total of 66 segments. For Group 2, every patient received both PLIF and BioFlex fixation, with 38 cases of one-segment, 16 of two-segment, and three of three-segment fixation (Tables 2 and 3 ).

Change of ROM. In terms of pre- and postoperative disc ROM, the patients in both Groups 1 and 2 in whom PLIF was performed in the diseased segments underwent the procedure regardless of whether the primary lesions were accompanied by instability. For Group 1 patients, the 66 segments treated with BioFlex dynamic stabilization only showed a change of ROM from $10.0 \pm 4.3^{\circ}$ (preoperatively) to $4.1 \pm 1.9^{\circ}$ (postoperatively; $\mathrm{p}=0.001$ ). This means that the BioFlex system allows stabilization and motion preservation of each segment. In comparing the ROM of the adjacent segments between groups, the segments in Group 1 patients showed smaller increases in ROM than did segments in Group 2 (Table 4). This tells us that the BioFlex system can correct instability and results in less degeneration of adjacent segments than the rigid fixation system.

Lumbosacral Angle. In Group 1, the lumbosacral angle was measured before and after the operation by using lateral x-ray films, with the lumbosacral angle defined as the angle between the lines penetrating the centroids of L-5 and L-3, and L-5 and L-1. Patients were classified into three groups according to the lumbosacral angle: those with kyphosis (Group A, angle $<150^{\circ}$ ); patients with angles greater than $150^{\circ}$ and less than $160^{\circ}$ (Group B), and those with flatback syndromes (Group $\mathrm{C}$, angle $\geq 160^{\circ}$ ). We checked angles preoperatively and on the day immediately postoperatively, and then every 3 months thereafter (Fig. 7). For Group A, the mean preoperative angle was $140.54 \pm 6.14^{\circ}$, whereas the mean angle at the last follow-up visit was $150.71 \pm 4.93^{\circ}$. For Group B, the mean preoperative angle was $154.85 \pm 2.69^{\circ}$, and the last follow-up angle was $156.98 \pm 5.90^{\circ}$. For Group C, the mean preoperative angle was $166.54 \pm 4.96^{\circ}$, and the last follow-up angle was $157.67 \pm 2.53^{\circ}$. In other words, the BioFlex system does not bend the rod to correct lordosis. From these results it appears that the BioFlex system can correct lordosis by itself, gradually and naturally.

Clinical Outcomes. During clinical evaluation, based on

TABLE 2

Level of BioFlex fixation with or without PLIF

\begin{tabular}{ccc}
\hline \hline & \multicolumn{2}{c}{ No. of Patients (\%) } \\
\cline { 2 - 3 } Op Level & Group 1 & Group 2 \\
\hline 1 segment & $28(60.9)$ & $38(66.7)$ \\
2 segments & $16(34.8)$ & $16(28.1)$ \\
3 segments & $2(4.3)$ & $3(5.3)$ \\
total & $46(100.0)$ & $57(100.0)$ \\
\hline
\end{tabular}

TABLE 3

Levels of PLIF bridged with Profix cage

\begin{tabular}{lccc}
\hline \hline & \multicolumn{3}{c}{ No. of Levels } \\
\cline { 2 - 4 } Cage Type & Group 1 & Group 2 & Total \\
\hline expandable & 8 & 28 & 36 \\
cylindrical & 23 & 51 & 74 \\
total & 31 & 79 & 110 \\
\hline
\end{tabular}

the visual analog scale, Group 1 reported better improvement of back pain than Group 2, with no significant difference in improvement of leg pain between the groups (Table 5). According to the Oswestry Disability Index scores, symptoms improved from $35.2 \pm 6.4$ (preoperatively) to $12.1 \pm 4.5$ (final follow-up visit) in Group 1 , and from $37.8 \pm 5.7$ to $13.6 \pm 4.2$ in Group 2 .

Complications. Among the 110 PLIF-treated segments in Groups 1 and 2, there was cage retropulsion on one level (but no clinical symptoms), halos were observed on three levels in the final follow-up review, and in eight levels instability was measured, all of which equates to a fusion rate of $90.0 \%$. One patient had a screw fracture at the 3month postoperative check-up and is currently under observation ( 7 months postoperatively) with no complaints. In one patient a loosening of the screw housing cap occurred, which we found on the routine postoperative check-up on the 7th day of the follow-up period and retightened, and currently the patient has no other problem.

A patient with degenerative spondylolisthesis of L-4 on L-5 was treated surgically with PLIF and BioFlex implantation at this level. This patient suffered a degenerative change in the adjacent segment 1 year after the operation, presenting with severe back and leg pain, and had to have an L3/4 PLIF and BioFlex fixation that extended from L2 to L-3 and L-4 (Fig. 8).

\section{Shape Memory Loop}

Surgical Methods. These data are summarized in Table 6. Among 194 patients, in $176(90.7 \%)$ memory loops were applied from the upper to the lower transitional segment, including the PLIF at the main diseased segments. We used memory loops alone in selected cases (18 patients [9.3\%]), for the correction of kyphoscoliosis after osteoporotic compression fractures in seven, for adjacentsegment instability after hard fixation in six, and for decompressive laminectomy without the need for discectomy in five.

Change in ROM Within Looped Segments. We used the paired-samples t-test for evaluation of the change in disc

TABLE 4

Adjacent-segment ROM

\begin{tabular}{cccc}
\hline \hline Group & Preop ROM $\left(^{\circ}\right)$ & Postop ROM $\left(^{\circ}\right)$ & p Value \\
\hline 1 & $8.4 \pm 3.4$ & $10.7 \pm 3.2$ & 0.001 \\
2 & $6.5 \pm 3.2$ & $10.5 \pm 4.6$ & 0.001 \\
\hline
\end{tabular}


TABLE 5

Visual analog scale score for low-back and leg pain

\begin{tabular}{llllll}
\hline \hline \multirow{2}{*}{ Pain } & \multicolumn{2}{c}{ Group 1 } & & \multicolumn{2}{c}{ Group 2 } \\
\cline { 2 - 3 } \cline { 5 - 6 } \cline { 5 - 6 } low-back & Preop & Postop & & Preop & Postop \\
\hline leg & $7.3 \pm 3.1$ & $1.4 \pm 1.8$ & & $7.4 \pm 2.4$ & $2.1 \pm 2.3$ \\
& $7.4 \pm 2.6$ & $1.3 \pm 1.6$ & & $7.5 \pm 1.6$ & $1.4 \pm 2.1$ \\
\hline
\end{tabular}

ROM before and 1 year after memory loop dynamic stabilization. These data are summarized in Table 7 . The ROM of the disc was significantly reduced when calculations involved the PLIF levels. There was no significant difference between the preoperative ROM of the disc and postoperative 1-year follow-up data at memory loops on the non-PLIF level. This means that memory loops affect the tight immobilization to the PLIF level and do not restrict the motion of the laminectomy with or without discectomy within the level at which the loop is applied.

Change in ROM in Adjacent Level Beyond Looped Segments. These data are summarized in Table 7. The ROM of the disc at one level above or below the segments fixed with memory loops increased slightly, but there was no statistical significance.

Change in Kyphosis. Two or four memory loops were applied at different levels for the correction of degenerative kyphoscoliosis or kyphosis and back pain occurring after osteoporotic compression fractures. Seven patients underwent surgery. The kyphotic angle was reduced from $23.96 \pm 11.7^{\circ}$ preoperatively to $16.25 \pm 12.34^{\circ}$ at 1 year postoperatively. A paired-sample t-test was done and the probability value was 0.007 .

Our illustrative case shows the correction of kyphosis (Fig. 6). A patient with an old L-1 compression fracture and a history of back pain and kyphosis underwent surgery in which two pairs of memory loops were used. The preoperative kyphotic angle between T-11 and L-2 was $32.5^{\circ}$ on the standing lateral $\mathrm{x}$-ray films. One year postoperatively the kyphotic angle was $22.5^{\circ}$.

Clinical Outcomes. The postoperative success rate was measured using the Prolo scale. Among 194 patients, excellent results were obtained in 55, good in 121, fair in 12, and poor in six. The overall success rate (excellent and good results) was $90.7 \%$.

Complications. During the 1-year follow-up period, there was no neurological worsening. Hardware failures

TABLE 6

Methods of implanting Nitinol shape memory loops in 194 patients

\begin{tabular}{cc}
\hline \hline \multicolumn{1}{c}{ Op Procedure } & No. of Patients \\
\hline PLIF + loop & 14 \\
1-level PLIF + 1-level loop & 120 \\
1-level PLIF + $\geq 2$-level loop & 16 \\
2-level PLIF + 2-level loop & 26 \\
2-level PLIF + $\geq 3$-level loop & \\
loop only & 5 \\
after decompressive laminectomy & 6 \\
treatment for adjacent-segment instability & 7 \\
correction of kyphoscoliosis & \\
\hline
\end{tabular}

occurred in four of 194 patients: these consisted of pullout of memory loops and memory loop fracture in two patients each. Cage-related complications did not occur. Nonfusion (indicated by a halo around the cage), more than $5^{\circ}$ movement on flexion and extension x-ray films obtained at the PLIF level, and cage retropulsion or displacement did not occur.

\section{Discussion}

Clinical experience indicates that a more rigid spinal stabilization system increases the risk of complications, including mechanical failure, device-related osteoporosis, and accelerated degeneration at adjoining levels. To avoid these complications and concurrently obtain adequate immobilization, the achievement of ideal stiffness is important. For that reason, the use of dynamic stabilization device systems would be a notable advance. ${ }^{16}$ Generally, the hard fixation system has been one of the most popular devices for rigid stabilization, although it has the critical disadvantage of lack of flexibility. Consequently, the soft or dynamic stabilization system has been introduced recently for the purpose of achieving more physiological stabilization.

Stabilization of the lumbar spine without fusion has been practiced sporadically during the last decade in Europe and East Asia. ${ }^{11}$ The demand for an ideal dynamic stabilization system is greater for younger patients with multisegment disc degeneration in whom adjacent-segment disease may be more likely to occur in the long-term follow-up period after fusion. Recently there has been an upsurge of interest in dynamic stabilization, with a further extension of indications to "topping off" an adjacent segment or stabilizing a segment following discectomy. ${ }^{16} \mathrm{~A}$ number of dynamic stabilization systems have been used clinically.

TABLE 7

Change in $\mathrm{ROM}^{*}$

\begin{tabular}{|c|c|c|c|c|}
\hline Level & $\begin{array}{c}\text { No. of } \\
\text { Segments }\end{array}$ & Preop ROM $\left(^{\circ}\right)$ & $\mathrm{ROM}$ at $1 \mathrm{Yr}\left({ }^{\circ}\right)$ & $\mathrm{p}$ Value \\
\hline \multicolumn{5}{|c|}{ within looped segments, including PLIF } \\
\hline $\mathrm{L} 1 / 2$ & 4 & $2.78 \pm 2.23$ & $3.59 \pm 2.68$ & 0.601 \\
\hline $\mathrm{L} 2 / 3$ & 44 & $4.98 \pm 3.13$ & $4.04 \pm 3.42$ & 0.084 \\
\hline $\mathrm{L} 3 / 4$ & 137 & $5.06 \pm 3.20$ & $3.39 \pm 3.83$ & 0.001 \\
\hline $\mathrm{L} 4 / 5$ & 156 & $5.57 \pm 3.59$ & $0.56 \pm 2.01$ & 0.001 \\
\hline L5/S1 & 0 & NA & NA & NA \\
\hline total & 341 & $5.26 \pm 3.38$ & $2.18 \pm 3.39$ & 0.001 \\
\hline \multicolumn{5}{|c|}{ within looped segments, excluding PLIF } \\
\hline $\mathrm{L} 1 / 2$ & 4 & $2.78 \pm 2.23$ & $3.59 \pm 2.68$ & 0.601 \\
\hline $\mathrm{L} 2 / 3$ & 38 & $5.14 \pm 3.22$ & $4.68 \pm 3.24$ & 0.412 \\
\hline $\mathrm{L} 3 / 4$ & 88 & $5.28 \pm 3.18$ & $5.28 \pm 3.58$ & 0.988 \\
\hline $\mathrm{L} 4 / 5$ & 15 & $3.96 \pm 2.38$ & $5.81 \pm 3.45$ & 0.192 \\
\hline L5/S1 & 0 & NA & NA & NA \\
\hline total & 145 & $5.04 \pm 3.12$ & $5.13 \pm 3.46$ & 0.807 \\
\hline \multicolumn{5}{|c|}{ one adjacent level beyond looped segment } \\
\hline $\mathrm{T} 12 / \mathrm{L} 1$ & 5 & $2.22 \pm 1.50$ & $2.34 \pm 1.38$ & 0.904 \\
\hline $\mathrm{L} 1 / 2$ & 32 & $3.62 \pm 2.50$ & $4.13 \pm 2.71$ & 0.402 \\
\hline $\mathrm{L} 2 / 3$ & 94 & $4.52 \pm 2.65$ & $5.03 \pm 2.88$ & 0.152 \\
\hline $\mathrm{L} 3 / 4$ & 10 & $4.99 \pm 1.89$ & $4.12 \pm 2.81$ & 0.467 \\
\hline $\mathrm{L} 4 / 5$ & 2 & $3.66 \pm 2.78$ & $8.68 \pm 1.22$ & 0.326 \\
\hline L5/S1 & 129 & $5.00 \pm 3.22$ & $5.09 \pm 3.83$ & 0.803 \\
\hline total & 272 & $4.61 \pm 2.92$ & $4.90 \pm 3.36$ & 0.215 \\
\hline
\end{tabular}

* NA = not applicable. 


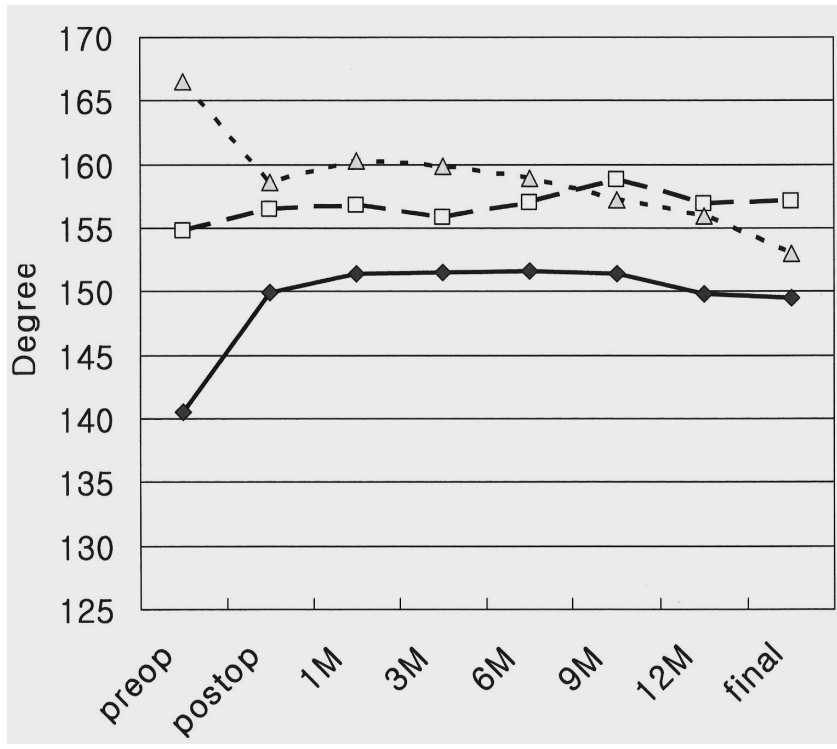

FIG. 7. Graph showing the change in the lumbosacral angle. Group A (diamonds) included patients whose spines were in a kyphotic state preoperatively (lumbosacral angle $<150^{\circ}$ ); patients in Group B (squares) had normal curvature preoperatively $\left(150^{\circ}<\right.$ lumbosacral angle $<160^{\circ}$ ); and those in Group $\mathrm{C}$ (triangles) had flatback syndrome (lumbosacral angle $>160^{\circ}$ ). At the final follow-up visit, lumbosacral angles in all three groups had converged at a normal curvature angle. $\mathrm{M}=\operatorname{month}(\mathrm{s})$.

\section{Nitinol Memory Loop}

Nitinol, a shape memory alloy of nickel and titanium, is flexible at low temperatures and regains its original shape when heated. ${ }^{6}$ Some spine surgeons have focused on this characteristic and have tried to apply it to correction of scoliosis. ${ }^{1,13,14,19}$

The KIMPF-DI fixing system has five types. ${ }^{5}$ We have used only one, the loop-fixing type. The mechanical characteristics of this type are similar to those of the interspinous ligaments (Fig. 9).,56 This system has unique temperature characteristics. Preliminary deformation is done at temperatures not exceeding $10^{\circ} \mathrm{C}$. The deformed shape is kept unchanged up to $26^{\circ} \mathrm{C}$, and shape recovery occurs after the material is heated up to $35^{\circ} \mathrm{C} .^{5}$ If this system is implanted in the human body with a cooled and deformed shape, it will regain its original shape spontaneously and fit snugly. On the other hand, Wever et al. ${ }^{19}$ experimented on scoliosis correction with Nitinol characterized by a shape memory recovering effect at a temperature of $50^{\circ} \mathrm{C}$. This temperature may be hazardous to the animal or human during the shape recovery process.

The key properties of Nitinol are as follows: 1) large forces that can be generated due to the shape memory effect; 2) excellent damping properties below the transition temperature; 3) excellent corrosion resistance; 4) nonmagnetic material; 5) high fatigue strength; 6) moderate impact resistance; 7) moderate heat resistance; and 8) biocompatibility. ${ }^{10}$ According to the International Organization of Standardization, the shape memory nickel-titanium alloy provoked no cytotoxic, allergic, or genotoxic responses. ${ }^{19}$ We used the Nitinol memory loop as the pos-

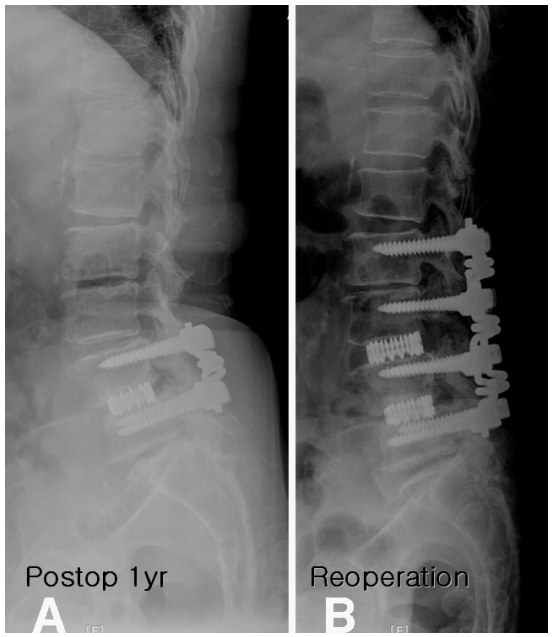

FIG. 8. Follow-up x-ray films obtained in a 67-year-old woman who had undergone surgery for degenerative spondylolisthesis at L4-5; $360^{\circ}$ fusion with PLIF and BioFlex had been performed. The topping off phenomenon developed 1 year postoperatively, and a repeated operation was performed; PLIF on L3/4 and extension of the BioFlex system from L-2 to L-3 and L-4.

terior column supporter or posterior tension bands for the aforementioned reasons.

The biomechanical similarity between the loop-fixing type of stabilization system and the interspinous ligaments provided preservation of motion at the posterior column that had undergone resection by laminectomy and removal of interspinous ligament; the ROM matched that in the intact posterior column. In our results, loop fixation at the resected posterior column provided the same ROM as that measured preoperatively, but did not provoke adjacent-segment hypermotion. This means that aggravation of adjacent-segment degeneration may not occur when the memory loop is applied at the PLIF or decompressive laminectomy area.

Ease of application on account of its heat-dependent plasticity and its recovery of the original shape produced many advantages, including a short operating time, less

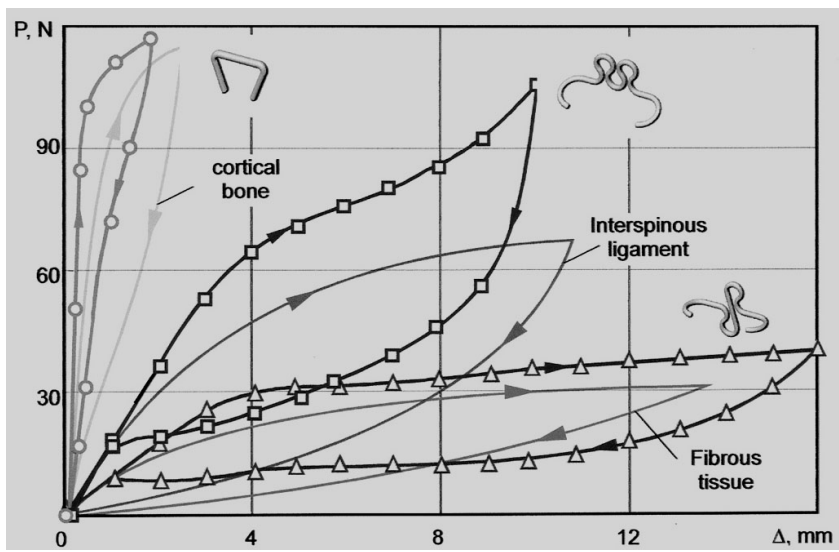

FIG. 9. Graph showing mechanical behavior of human body tissue and various shape memory loop fixing devices. Mechanical behavior of the memory loop is similar to that of the interspinous ligament. $\mathrm{N}=$ Newtons; $\mathrm{P}=$ power. 
bleeding, and elimination of the need for wide muscle dissection for pedicle screw fixation.

There were no PLIF-related problems in our series. This might be due to the tension band effect of the flexible loop. The looped shape might transmit the elastic and tensile load to the vertebra-cage-vertebra structure. This stress can enhance the fusion rate of the PLIF level.

Two cases of pullout and two cases of memory loop fracture occurred in the earliest surgeries. At that time, we did not understand the proper size measurement of the loop. Pullout occurred because of the loose fit with a larger loop, and loop fracture occurred because of the tight fit with a smaller loop. Now, we always use the $1.5-\mathrm{cm}$ smaller loop in two-lamina applications and the 2-cm smaller loop when applying it at three levels.

We have also used the loop in kyphosis correction for disorders such as osteoporotic compression fractures. Osteoporotic bone did not cause the hardware failure or loosening; this might be due to the elastic and tensile property of the loop. Some degree of correction of kyphoscoliosis could be achieved using this memory loop. According to our data, improvement in kyphosis was not satisfactory and not statistically significant, but kyphosis was still somewhat improved $(\mathrm{p}=0.007)$. Unsatisfactory recovery of kyphosis might be due to continuous microfractures caused by compression of osteoporotic vertebrae. Long-term follow-up review and analysis is required in these cases.

\section{BioFlex System}

The BioFlex system consists of titanium screws and 4$\mathrm{mm}$ Nitinol rods. The rod's coiling consists of one and two turns, and due to the mechanical properties of Nitinol and the rod's shape, these devices possess superelasticity and rigidity, and therefore act as a tension band at the posterior spinal columns.

Compared with the rigid fixation, dynamic stabilization should permit continuous movement. An ideal system should consist of rods with no fatigue failure, and there should be no loosening between the device and bone junction. Because of these concerns, many biomechanical tests were conducted using the BioFlex system. No particular strains were imposed on the device during the fatigue strength test (American Society for Testing and Materials F1717) of 9-mm displacement with 20 million vibrations at $5 \mathrm{~Hz}$, and the compression, torsion, and tension tests did not transmit much strain to the device. A test of the biocompatibility of the BioFlex rod was also performed. A nickel extraction test using inductively coupled plasmaatomic emission spectroscopy was conducted for 9 days with no signs of extraction, and a galvanic corrosion test performed to assess the interaction between other metal contacts revealed no noticeable effects, which led to a conclusion that the BioFlex rod is safe for the body.

Since the introduction of the Graf soft-stabilization system in $1988,{ }^{2}$ many more such devices have been invented. The disadvantage of the Graf fixation, however, is that the ligament flavum and the joint capsules collapse inwardly, which leads to the narrowing of the neural foramen and spinal canal, exerting compression on the nerve root and inflicting leg pain. These problems occurred more frequently when the disc space was reduced due to degenerative lumbar disc disorder or laminectomy, or when patients underwent simple discectomy. Also, there is a possibility of lateral compression on the nerve root when the upper vertebral body is displaced posteriorly at the fixed segment of the Graf band. Also, although this fixation treatment may have corrected the posterior instability, it could not correct the anterior- and middle-column instability inflicted by degenerative lumbar disorders. ${ }^{7}$

The Dynesys system (Zimmer Spine) was developed later to overcome the disadvantage of the Graf system. ${ }^{18}$ The Dynesys system inserts a plastic tube into the Graf tension band to prevent overextension, which was a disadvantage of the earlier system. Therefore, during flexion the tension band restricts the motion, and during extension the tension band partially bears the weight and constrains the motion. Nevertheless, biomechanical studies revealed that during flexion and extension this system displayed 1 to $3^{\circ}$ more movement than the rigid system, but when compared with the intact spine, the motion range during extension was similar, whereas during flexion approximately $30 \%$ restriction occurred. ${ }^{15}$ In a recent report on a 2-year follow-up study of 31 patients, Grob et al. ${ }^{3}$ related that only $67 \%$ of the patients showed improved symptoms of back pain, $50 \%$ affirmed an increased quality of life, and $19 \%$ needed a repeated operation during the follow-up period.

Unlike the Graf, the BioFlex system prevents excessive lordosis during extension, thereby maintaining physiological ROM during flexion and extension and consequently improving the symptoms. The BioFlex dynamic stabilization system allows modification of the segmental neutral angle and disc height because it is a pedicle screw-based system. Thus, modification is possible through the compression or distraction of the two screws after inserting the rods and before final fixation.

Also, the BioFlex screw head has two grooves, which enable insertion of two rods per segment, and which is a strong point of this system. The BioFlex system is designed to use one rod per segment, so if the surgeon needs to operate on multiple segments, the number of rods to be used increases accordingly. Due to this design and the flexibility and elasticity of the rod, there is no need to contour the device to fit the natural sagittal curve of the spine. As can be seen in the Results section, the action of the BioFlex system and the soft tissues (muscle, ligament, and so on) react with each other to achieve a natural lordosis angle in the lumbar spine. Even though topping-off or bottoming-off phenomena appeared after hard fixation with the BioFlex system (Fig. 8), an easy repeat operation and extension of BioFlex soft fixation can be achieved without a long incision, by including the whole scar from the previous operation.

According to Wolff's law, ${ }^{17}$ to improve the fusion rate of an interbody fusion device, it is better to increase the stress between the graft and vertebral body rather than perform a rigid pedicle screw fixation. Using this system with PLIF can also enhance the fusion rate at the main diseased level.

Kim has claimed the need for "combined fixation" since 1997. Many patients need soft fixation to prevent adjacent-segment degeneration at the transitional segment, which is the upper level in which fusion is needed. ${ }^{4,8}$ This is why the Graf system was used, and afterward, the 
Nitinol shape memory loop and BioFlex system. As a dynamic stabilization system, BioFlex and the shape memory loop also achieve fusion by using PLIF at the main diseased segment, and this procedure prevents adjacent-segment degeneration and enhances the fusion rate of PLIF.

This "combined fixation" can incorporate the two main themes of spine surgery, that is, hard immobilization and preservation of motion.

\section{Conclusions}

We have used the Nitinol spring rod dynamic stabilization system (BioFlex) and Nitinol memory loops (KIMPF-DI Fixing System) as posterior dynamic stabilizers in degenerative lumbar diseases for the last 2 years, and we have analyzed short-term follow-up results.

The BioFlex system and the Nitinol shape memory loop posterior dynamic stabilization system seem to be a flexible and adequate posterior column supporter acting as the posterior ligamentous structures. These semirigid fixation systems are more physiologically compatible than hard fixation and can reduce postoperative adjacent-segment problems. Another advantage of the BioFlex system is that it can realign the normal sagittal balance even if the patient's preoperative lumbosacral angle was kyphotic or hyperlordotic (flatback syndrome). The other advantage of the Nitinol memory loop was easy application, lessened tissue damage, some correction of kyphosis, and no major complications.

Combining PLIF at the main lesion level with the BioFlex system or memory loop around the transitional segments will enhance the fusion rate, reduce the adjacent-segment degeneration, and provide stabilized motion.

\section{Disclaimer}

None of the authors have a financial interest in any of the devices or procedures discussed in this paper.

\section{References}

1. Braun JT, Ogilvie JW, Akyuz E, Brodke DS, Bachus KN: Fusionless scoliosis correction using a shape memory alloy staple in the anterior thoracic spine of the immature goat. Spine 29: 1980-1989, 2004

2. Graf H: [Lumbar instability surgical treatment without fusion. Soft system stabilization.] Rachis 412:123-137, 1992 (Fr)

3. Grob D, Benini A, Junge A, Mannion AF: Clinical experience with the Dynesys semirigid fixation system for the lumbar spine: surgical and patient-oriented outcome in 50 cases after an average of 2 years. Spine 30:324-331, 2005

4. Ha Y, Kim YS, Yoon DH, Jin DK, Park HW: [Graf soft fixation for the treatment of degenerative lumbar disease.]
J Korean Neurosurg Soc 27:1370-1378, 1998 (Korean)

5. Il'in AA, Kollerov MY, Khachin VI, Gusev DA: Medical instruments and implants of titanium nickelide: physical metallurgy, technology, and application. Russian Metallurgy (Metally) 3:296-300, 2002

6. Il' in AA, Kollerov MJ, Sergeyv SV, Zagorodnyj NV, Radnaeyv EB, Nevzorov AM, et al: [Biologically and mechanically compatible implants from titanium nickelide in the treatment of damaged dorsal and lumbar spine.] The Bulletin of Traumatology and Orthopedics 2:19-26, 2002 (Russian)

7. Kim YS, Chin DK: Graf soft stabilization: Graf ligamentoplasty, in Kim DH, Cammisa FP Jr, Fessler RG (eds): Dynamic Reconstruction of the Spine. New York: Thieme, 2006, pp 305-311

8. Kim YS, Cho YE, Jin BH, Chin DK, Yoon DH: [Soft Graf fixation and posterior lumbar interbody fusion in multiple degenerative lumbar diseases.] J Korean Neurosurg Soc 27: 229-236, 1998 (Korean)

9. Kim YS, Moon BJ: Bioflex spring rod pedicle screw system, in Kim DH, Cammisa FP Jr, Fessler RG (eds): Dynamic Reconstruction of the Spine. New York: Thieme, 2006, pp 340-346

10. Kim YS, Zhang HY: Shape memory implant (KIMPF-DI fixing) system, in Kim DH, Cammisa FP Jr, Fessler RG (eds): Dynamic Reconstruction of the Spine. New York: Thieme, 2006, pp 292-298

11. Mulholland RC, Sengupta DK: Rationale, principles and experimental evaluation of the concept of soft stabilization. Eur Spine J 11 (2 Suppl): S198-S205, 2002

12. Prolo DJ, Oklund SA, Butcher M: Toward uniformity in evaluating results of lumbar spine operations. A paradigm applied to posterior lumbar interbody fusion. Spine 11:601-606, 1986

13. Sanders JO, Sanders AE, More R, Ashman RB: A preliminary investigation of shape memory alloys in the surgical correction of scoliosis. Spine 18:1640-1646, 1993

14. Schmerling MA, Wilkov MA, Sanders AE, Woosley JE: Using the shape recovery of nitinol in the Harrington rod treatment of scoliosis. J Biomed Mater Res 10:879-892, 1976

15. Schmoelz W, Huber JF, Nydegger T, Dipl-Ing, Claes L, Wilke HJ: Dynamic stabilization of the lumbar spine and its effects on adjacent segments: an in vitro experiment. J Spinal Disord Tech 16:418-423, 2003

16. Sengupta DK: Dynamic stabilization devices in the treatment of low back pain. Orthop Clin North Am 35:43-56, 2004

17. Smit TH, Odgaard A, Schneider E: Structure and function of vertebral trabecular bone. Spine 22:2823-2833, 1997

18. Stoll TM, Dubois G, Schwarzenbach O: The dynamic neutralization system for the spine: a multi-center study of a novel non-fusion system. Eur Spine J 11 (2 Suppl): S170-S178, 2002

19. Wever DJ, Elstrodt JA, Veldhuizen AG, v Horn JR: Scoliosis correction with shape-memory metal: results of an experimental study. Eur Spine J 11:100-106, 2002

Manuscript submitted October 20, 2006.

Accepted November 20, 2006.

Address reprint requests to: Young-Soo Kim, M.D, Ph.D., Kwang-Hye Hospital, 995-25, Daechi-dong, Kangnam-gu, Seoul, Korea, 135-280. email: lamikimys@ hanmail.net. 